Wei Gao*, Yan-Jie Bai, Jia-Le Mao and Xue-Quan Guo

\title{
The crystal structure of pentakis(carbonyl)- $\{\mu$-[2,3-bis(sulfanyl)propan-1-olato]\}- (triphenylphosphane)diiron ( $\mathrm{Fe}-\mathrm{Fe}$ ) $\mathrm{C}_{26} \mathrm{H}_{21} \mathrm{Fe}_{2} \mathrm{O}_{6} \mathrm{PS}_{2}$
}

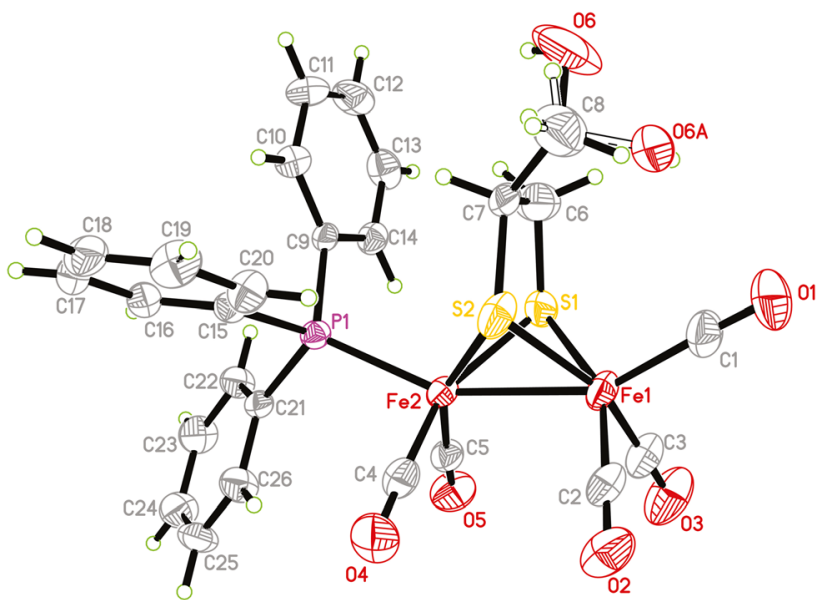

https://doi.org/10.1515/ncrs-2021-0003

Received January 5, 2021; accepted January 22, 2021;

published online February 8, 2021

\begin{abstract}
$\mathrm{C}_{26} \mathrm{H}_{21} \mathrm{Fe}_{2} \mathrm{O}_{6} \mathrm{PS}_{2}$, monoclinic, $P 2_{1} / c$ (no. 14), $a=9.0892(6) \AA$, $b=27.6631(18) \AA, c=11.3409(8) \AA, \beta=106.409(2)^{\circ}$, $V=2735.4(3) \AA^{3}, Z=4, R_{g t}(F)=0.0670, w R_{\text {ref }}\left(F^{2}\right)=0.1620$, $\mathrm{T}=296(2) \mathrm{K}$.
\end{abstract}

CCDC no.: 2057958

The molecular structure is shown in the figure. Table 1 contains crystallographic data and Table 2 contains the list of the atoms including atomic coordinates and displacement parameters.

*Corresponding author: Wei Gao, School of Pharmacy, Henan University of Chinese Medicine, Zhengzhou, Henan 450046, P. R. China, E-mail: weigao415@aliyun.com. https://orcid.org/00000002-2998-9487

Yan-Jie Bai and Jia-Le Mao, School of Pharmacy, Henan University of Chinese Medicine, Zhengzhou, Henan 450046, P. R. China Xue-Quan Guo, Zhengzhou Second Hospital, Zhengzhou, Henan 450046, P. R. China
Table 1: Data collection and handling.

\begin{tabular}{ll}
\hline Crystal: & Red block \\
Size: & $0.32 \times 0.24 \times 0.22 \mathrm{~mm}$ \\
Wavelength: & Mo $K \alpha$ radiation $(0.71073 \AA)$ \\
$\mu:$ & $1.31 \mathrm{~mm}^{-1}$ \\
Diffractometer, scan mode: & Bruker D8 QUEST, $\varphi$ and $\omega$ \\
$\theta_{\text {max }}$, completeness: & $25.1^{\circ},>99 \%$ \\
$N(h k l)_{\text {measured }}, N(h k l)_{\text {unique }}, R_{\text {int }}:$ & $63350,4837,0.090$ \\
Criterion for $I_{\text {obs }}, N(h k l)_{\text {gt }}:$ & $I_{\text {obs }}>2 \sigma\left(I_{\text {obs }}\right), 3418$ \\
$N(\text { param })_{\text {refined }}:$ & 344 \\
Programs: & Bruker [1], Olex2 [2], SHELX [3, 4] \\
\hline
\end{tabular}

\section{Source of material}

A mixture of complex $\left[\mathrm{Fe}_{2}(\mathrm{CO})_{6}\left\{\mu-\mathrm{SCH}_{2} \mathrm{CH}\left(\mathrm{CH}_{2} \mathrm{OH}\right)\right\}\right]$ (1 mmol) and triphenylphosphine (1 mmol) was treated with $\mathrm{Me}_{3} \mathrm{NO} \cdot 2 \mathrm{H}_{2} \mathrm{O}(1 \mathrm{mmol})$. The resulting solution was stirred at room temperature for $0.5 \mathrm{~h}$. Afterwards, the solvent was reduced in vacuo and the residue was subjected to TLC separation using $\mathrm{CH}_{2} \mathrm{Cl}_{2}$ : petroleum ether $=1: 3(\mathrm{v} / \mathrm{v})$ as eluent. The title complex was obtained from the main red band. Slow evaporation of $\mathrm{CH}_{2} \mathrm{Cl}_{2} /$ hexane solution at $4{ }^{\circ} \mathrm{C}$ afforded crystals suitable for $\mathrm{X}$-ray diffraction analysis.

\section{Experimental details}

The structure was solved by direct method with the SHELXS program. Hydrogen atoms were positioned geometrically $(\mathrm{C}-\mathrm{H}=0.93-0.98 \AA$ and $\mathrm{O}-\mathrm{H}=0.82 \AA)$. Their $U_{\text {iso }}$ values were set to $1.2 U_{e q}$ or $1.5 U_{e q}$ of the parent atoms. The hydroxy group features a disorder (see the figure).

\section{Comment}

Over the past decades, dithiolate-bridging diiron complexes have attracted great interest due to their structural resemblance with the active site of [FeFe]-hydrogenases [5-7]. [FeFe]-hydrogenases are natural metalloenzymes that can catalyze the reduction of protons to $\mathrm{H}_{2}[8,9]$. The 
Table 2: Fractional atomic coordinates and isotropic or equivalent isotropic displacement parameters $\left(\AA^{2}\right)$.

\begin{tabular}{|c|c|c|c|c|}
\hline Atom & $x$ & $y$ & $z$ & $U_{\text {iso }}{ }^{*} / U_{\text {eq }}$ \\
\hline Fe1 & $0.15445(10)$ & $0.47736(3)$ & $0.78454(10)$ & 0.0539 (3) \\
\hline $\mathrm{Fe} 2$ & $0.04267(9)$ & $0.39648(3)$ & $0.80761(8)$ & $0.0408(2)$ \\
\hline S1 & $0.0102(2)$ & $0.43417(6)$ & $0.62538(18)$ & $0.0630(5)$ \\
\hline S2 & $-0.0601(2)$ & $0.46758(6)$ & $0.8423(2)$ & $0.0666(6)$ \\
\hline P1 & $-0.14211(17)$ & $0.34028(5)$ & 0.77659 (14) & 0.0377 (4) \\
\hline 01 & $0.1284(9)$ & $0.5793(2)$ & $0.7129(7)$ & $0.116(2)$ \\
\hline 02 & $0.3498(7)$ & $0.4877(3)$ & $1.0349(6)$ & $0.095(2)$ \\
\hline 03 & $0.4231(7)$ & $0.4501(3)$ & $0.7076(7)$ & $0.110(2)$ \\
\hline 04 & $0.1733(7)$ & $0.3814(2)$ & $1.0694(5)$ & 0.0915 (19) \\
\hline 05 & $0.2586(6)$ & $0.3345(2)$ & $0.7345(5)$ & $0.0789(16)$ \\
\hline $06^{\mathrm{a}}$ & $-0.3938(18)$ & $0.5321(5)$ & $0.5693(15)$ & $0.177(9)$ \\
\hline$H 6^{\mathrm{a}}$ & -0.4199 & 0.5054 & 0.5395 & $0.265^{*}$ \\
\hline $06 A^{b}$ & $-0.190(2)$ & $0.5637(7)$ & $0.673(3)$ & 0.181 \\
\hline$H 6 A^{b}$ & -0.1504 & 0.5572 & 0.6184 & $0.271^{*}$ \\
\hline $\mathrm{C} 1$ & $0.1361(9)$ & $0.5396(3)$ & $0.7370(8)$ & $0.076(2)$ \\
\hline $\mathrm{C} 2$ & $0.2699(8)$ & $0.4846(3)$ & $0.9364(8)$ & $0.064(2)$ \\
\hline C3 & $0.3178(9)$ & $0.4613(3)$ & $0.7375(8)$ & $0.071(2)$ \\
\hline $\mathrm{C} 4$ & $0.1203(8)$ & $0.3869(3)$ & $0.9665(7)$ & $0.0583(18)$ \\
\hline $\mathrm{C} 5$ & $0.1747(7)$ & $0.3581(2)$ & $0.7667(6)$ & $0.0493(16)$ \\
\hline $\mathrm{C} 6$ & $-0.1707(10)$ & $0.4643(3)$ & $0.5850(9)$ & $0.087(3)$ \\
\hline H6B & -0.1638 & 0.4937 & 0.5401 & $0.104^{*}$ \\
\hline $\mathrm{H} 6 \mathrm{C}$ & -0.2476 & 0.4436 & 0.5319 & $0.104^{*}$ \\
\hline $\mathrm{C} 7$ & $-0.2161(8)$ & $0.4762(3)$ & $0.6950(10)$ & $0.083(3)$ \\
\hline $\mathrm{H} 7$ & -0.2984 & 0.4537 & 0.6975 & 0.099* \\
\hline $\mathrm{C} 8$ & $-0.2855(14)$ & $0.5268(4)$ & $0.6842(11)$ & $0.114(4)$ \\
\hline $\mathrm{H} 8 \mathrm{AA} \mathrm{A}^{\mathrm{a}}$ & -0.3343 & 0.5318 & 0.7490 & $0.137^{\star}$ \\
\hline$H 8 A B^{a}$ & -0.2052 & 0.5508 & 0.6935 & $0.137^{\star}$ \\
\hline $\mathrm{H} 8 \mathrm{BC}^{\mathrm{b}}$ & -0.3752 & 0.5274 & 0.6134 & $0.137^{*}$ \\
\hline $\mathrm{H} 8 \mathrm{BD}^{\mathrm{b}}$ & -0.3199 & 0.5328 & 0.7564 & $0.137^{*}$ \\
\hline C9 & $-0.2793(6)$ & 0.34205 (19) & $0.6247(5)$ & 0.0384 (13) \\
\hline C10 & $-0.4319(7)$ & $0.3516(2)$ & $0.6067(6)$ & $0.0518(16)$ \\
\hline $\mathrm{H} 10$ & -0.4697 & 0.3545 & 0.6745 & $0.062^{\star}$ \\
\hline C11 & $-0.5296(8)$ & $0.3569(3)$ & $0.4918(7)$ & $0.069(2)$ \\
\hline H11 & -0.6325 & 0.3638 & 0.4822 & $0.082^{*}$ \\
\hline C12 & $-0.4774(10)$ & $0.3522(3)$ & $0.3905(7)$ & $0.072(2)$ \\
\hline $\mathrm{H} 12$ & -0.5449 & 0.3555 & 0.3123 & $0.087^{\star}$ \\
\hline C13 & $-0.3266(9)$ & $0.3427(2)$ & $0.4041(6)$ & 0.0636 (19) \\
\hline H13 & -0.2912 & 0.3394 & 0.3352 & $0.076^{*}$ \\
\hline C14 & $-0.2258(7)$ & $0.3379(2)$ & $0.5203(6)$ & 0.0486 (15) \\
\hline H14 & -0.1225 & 0.3318 & 0.5294 & $0.058^{\star}$ \\
\hline C15 & $-0.2676(7)$ & $0.3375(2)$ & $0.8774(5)$ & $0.0426(14)$ \\
\hline C16 & $-0.3450(7)$ & $0.2951(3)$ & $0.8861(6)$ & 0.0557 (17) \\
\hline H16 & -0.3301 & 0.2679 & 0.8429 & $0.067^{*}$ \\
\hline C17 & $-0.4441(8)$ & $0.2930(3)$ & $0.9585(7)$ & $0.069(2)$ \\
\hline H17 & -0.4953 & 0.2643 & 0.9638 & $0.083^{*}$ \\
\hline C18 & $-0.4675(8)$ & $0.3328(3)$ & $1.0225(7)$ & $0.073(2)$ \\
\hline H18 & -0.5352 & 0.3313 & 1.0704 & $0.088^{\star}$ \\
\hline C19 & $-0.3904(9)$ & $0.3747(3)$ & $1.0156(7)$ & $0.076(2)$ \\
\hline H19 & -0.4057 & 0.4018 & 1.0592 & $0.092^{*}$ \\
\hline $\mathrm{C} 20$ & $-0.2896(8)$ & $0.3769(3)$ & $0.9438(6)$ & 0.0584 (18) \\
\hline $\mathrm{H} 2 \mathrm{O}$ & -0.2365 & 0.4054 & 0.9408 & $0.070^{*}$ \\
\hline $\mathrm{C} 21$ & $-0.0634(6)$ & $0.2792(2)$ & 0.7929 & 0.0399 (14) \\
\hline $\mathrm{C} 22$ & $-0.1010(8)$ & $0.2444(2)$ & $0.7017(6)$ & $0.0532(17)$ \\
\hline $\mathrm{H} 22$ & -0.1704 & 0.2518 & 0.6264 & $0.064^{\star}$ \\
\hline C23 & $-0.0365(9)$ & $0.1987(3)$ & $0.7211(7)$ & $0.068(2)$ \\
\hline
\end{tabular}

Table 2: (continued)

\begin{tabular}{lrrrr}
\hline Atom & $\boldsymbol{x}$ & $\boldsymbol{y}$ & $\boldsymbol{z}$ & $\boldsymbol{U}_{\text {iso }} \boldsymbol{U}_{\text {eq }}$ \\
\hline $\mathrm{H} 23$ & -0.0637 & 0.1757 & 0.6591 & $0.081^{*}$ \\
$\mathrm{C} 24$ & $0.0663(9)$ & $0.1872(3)$ & $0.8303(8)$ & $0.070(2)$ \\
$\mathrm{H} 24$ & 0.1092 & 0.1565 & 0.8428 & $0.084^{*}$ \\
$\mathrm{C} 25$ & $0.1060(8)$ & $0.2210(3)$ & $0.9210(7)$ & $0.067(2)$ \\
$\mathrm{H} 25$ & 0.1764 & 0.2133 & 0.9956 & $0.081^{*}$ \\
$\mathrm{C} 26$ & $0.0422(8)$ & $0.2668(2)$ & $0.9028(7)$ & $0.0588(18)$ \\
$\mathrm{H} 26$ & 0.0708 & 0.2896 & 0.9655 & $0.071^{*}$ \\
\hline
\end{tabular}

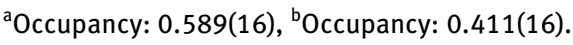

X-ray crystallographic structures of the active site of [FeFe]-hydrogenases have promoted chemists to produce a great number of diiron analogues as mimics for the active site of [FeFe]-hydrogenases [10-13]. In this study, we have successfully prepared a diiron analogue with a monophosphine ligand.

The asymmetric unit of the title complex consists of a butterfly diiron ethane-1,2-dithiolate dinuclear complex with five terminal carbonyls and a triphenylphosphine ligand, respectively. The phosphine ligand occupies an apical position of the distorted octahedral geometry of Fe2, in good agreement with monosubstituted analogues [14-16]. The Fe1-Fe2 bond length [2.5013(12) $\AA]$ is identical to that of the parent complex $\left[\mathrm{Fe}_{2}(\mathrm{CO})_{6}\left\{\mu-\mathrm{SCH}_{2} \mathrm{CH}\left(\mathrm{CH}_{2} \mathrm{OH}\right)\right\}\right][2.4998(6) \AA]$ [17], but much shorter than those in diphosphine-substituted analogues [18-20] as well as in natural [FeFe]-hydrogenases $[8,9]$. Note that the 06 atom is disordered over two sites with an occupancy of $0.589(16)$. The disorder suggests that it is unlikely that this $\mathrm{OH} \cdots \mathrm{O}$ hydrogen bond is present. Moreover, weak hydrogen bonds are also observed.

Author contribution: All the authors have accepted responsibility for the entire content of this submitted manuscript and approved submission.

Research funding: None declared.

Conflict of interest statement: The authors declare no conflicts of interest regarding this article.

\section{References}

1. BRUKER. SAINT, APEX2 and SADABS; Bruker AXS Inc.: Madison, Wisconsin, USA, 2009.

2. Dolomanov O. V., Bourhis L. J., Gildea R. J., Howard J. A. K., Puschmann H. OLEX2: a complete structure solution, refinement and analysis program. J. Appl. Crystallogr. 2009, 42, 339-341.

3. Sheldrick G. M. Crystal structure refinement with SHELXL. Acta Crystallogr. 2015, C71, 3-8.

4. Sheldrick G. M. A short history of SHELX. Acta Crystallogr. 2008, A64, 112-122. 
5. Tard C., Pickett C. J. Structural and functional analogues of the active sites of the [Fe]-, [NiFe]-, and [FeFe]-hydrogenases. Chem. Rev. 2009, 109, 2245-2274.

6. Li Y., Rauchfuss T. B. Synthesis of diiron(I) dithiolato carbonyl complexes. Chem. Rev. 2016, 116, 7043-7077.

7. Rauchfuss T. B. Diiron azadithiolates as models for the [FeFe]hydrogenase active site and paradigm for the role of the second coordination sphere. Acc. Chem. Res. 2015, 48, 2107-2116.

8. Peters J. W., Lanzilotta W. N., Lemon B. J., Seefeldt L. C. X-ray crystal structure of the Fe-only hydrogenase $(\mathrm{Cpl})$ from Clostridium pasteurianum to 1.8 angstrom resolution. Science 1998, 282, 1853-1857.

9. Nicolet Y., Piras C., Legrand P., Hatchikian C. E., Fontecilla- Camps J. C. Desulfovibrio Desulfuricans iron hydrogenase: the structure shows unusual coordination to an active site Fe binuclear center. Structure 1999, 7, 13-23.

10. Ghosh S., Hogarth G., Hollingsworth N., Holt K. B., Richard I., Richmond M. G., Sanchez B. E., Unwin D. Models of the iron-only hydrogenase: a comparison of chelate and bridge isomers of $\mathrm{Fe}_{2}(\mathrm{CO})_{4}\left\{\mathrm{Ph}_{2} \mathrm{PN}(\mathrm{R}) \mathrm{PPh}_{2}\right\}(\mu-\mathrm{pdt})$ as proton-reduction catalysts. Dalton. Trans. 2013, 42, 6775-6792.

11. Gloaguen F., Lawrence J. D., Rauchfuss T. B. Biomimetic hydrogen evolution catalyzed by an iron carbonyl thiolate. J. Am. Chem. Soc. 2001, 123, 9476-9477.

12. Lyon E. J., Georgakaki I. P., Reibenspies J. H., Darensbourg M. Y. Coordination sphere flexibility of active-site models for Fe-only hydrogenase: studies in intra- and intermolecular diatomic ligand exchange. J. Am. Chem. Soc. 2001, 123, 3268-3278.

13. Zhao P. H., Ma Z. Y., Hu M. Y., He J., Wang Y. Z., Jing X. B., Chen H. Y., Li Y. L. PNP-chelated and -bridged diiron dithiolate complexes $\mathrm{Fe}_{2}(\mu$-pdt $)(\mathrm{CO})_{4}\left\{\left(\mathrm{Ph}_{2} \mathrm{P}\right)_{2} \mathrm{NR}\right\}$ together with related monophosphine complexes for the $[2 \mathrm{Fe}]_{H}$ subsite of [FeFe]hydrogenases: preparation, structure, and electrocatalysis. Organometallics 2018, 37, 1280-1290.
14. Hu M. Y., Zhao P. H., Li J. R., Gu X. L., Jing X. B., Liu X. F. Synthesis, structures, and electrocatalytic properties of phosphinemonodentate, -chelate, and -bridge diiron 2,2-dimethylpropanedithiolate complexes related to [FeFe]hydrogenases. Appl. Organomet. Chem. 2020, 34, e5523.

15. Yan L., Hu K., Liu X. F., Li Y. L., Liu X. H., Jiang Z. Q. Diiron ethane1,2-dithiolate complexes with 1,2,3-thiadiazole moiety: synthesis, X-ray crystal structures, electrochemistry, and fungicidal activity. Appl. Organomet. Chem. 2021, 35, e6084.

16. Lü S., Huang H. L., Zhang R. F., Ma C. L., Li Q. L., He J., Yang J., Li T., Li Y. L. Phosphine-substituted Fe-Te clusters related to the active site of [FeFe]- $\mathrm{H}_{2}$ ases. Inorg. Chem. Front. 2020, 7, 2352-2361.

17. Niu S. J., Yu X. Y., Liu X. F., Li Y. L. Tris(2-methoxyphenyl) phosphine substituted diiron ethanedithiolate complexes containing hydroxymethyl, methyl or ethyl groups. Polyhedron 2017, 137, 127-133.

18. Zhao P. H., Hu M. Y., Ma Z. Y., Li J. R., Wang Y. Z., He J., Li Y. L., Liu $X . F$. Influence of dithiolate bridges on the structures and electrocatalytic performance of small bite-angle PNP-chelated diiron complexes $\mathrm{Fe}_{2}\left(\mu\right.$-xdt) $(\mathrm{CO})_{4}\left\{\mathrm{k}^{2}-\left(\mathrm{Ph}_{2} \mathrm{P}\right)_{2} \mathrm{NR}\right\}$ related to [FeFe]- hydrogenases. Organometallics 2019, 38, 385-394.

19. Hu M. Y., Yan L., Li J. R., Wang Y. H., Zhao P. H., Liu X. F. Reactions of $\mathrm{Fe}_{2}\left(\mu\right.$-odt) $(\mathrm{CO})_{6}$ (odt $=1$, 3-oxadithiolate) with small bite-angle diphosphines to afford the monodentate, chelate, and bridge diiron complexes: selective substitution, structures, protonation, and electrocatalytic proton reduction. Appl. Organomet. Chem. 2019, 33, e4949.

20. Zhao P. H., Hu M. Y., Li J. R., Wang Y. Z., Lu B. P., Han H. F., Liu X. F. Impacts of coordination modes (chelate versus bridge) of PNPdiphosphine ligands on the redox and electrocatalytic properties of diiron oxadithiolate complexes for proton reduction. Electrochim. Acta 2020, 353, 136615. 